\title{
Micro-systems for Optical Protein-Chip
}

\author{
Gang Jin, Zhan- Hui Wang
}

National Microgravity Laboratory, Institute of Mechanics, Chinese Academy of Sciences, Beijing 100080, China, E-mail: gajin@imech.ac.cn

\begin{abstract}
Protein-Chip as micro-assays for the determination of protein interaction, the analysis, the identification and the purification of proteins has large potential applications. The Optical Protein-Chip is able to detect the multi-interaction of proteins and multi-bio-activities of molecules directly and simultaneously with no labeling. The chip is a small matrix on solid substrate containing multi-micro-area prepared by microfabrication with photolithography or soft lithography for surface patterning, and processed with surface modification which includes the physical, chemical, and bio-chemical modifications, etc. The ligand immobilization, such as protein immobilization, especially the oriented immobilization with low steric hindrance and high bio-specific binding activity between ligand and receptor is used to form a sensing surface. Each area of the pattern is corresponding to only one bioactivity. The interval between the areas is non-bioactive and optically extinctive. The affinity between proteins is used to realize non-labeling microassays for the determination of protein identification and protein interaction. The sampling of the chip is nondisturbing, performed with imaging ellipsometry and image processing on a database of proteins.
\end{abstract}

Keywords: protein-chip, surface patterning, bio-affinity, imaging ellipsometry

\section{Introduction}

The micro-system for an optical Protein-Chip is demonstrated in this report, which is based on the concept of biosensor with imaging ellipsometry presented several years ago $[1,2]$. As an approach to the micro-system, the basic idea of the biosensor is repeated at the beginning.

The basic principle is that one kind of optical imaging technique - imaging ellipsometry is used to visualize optical biochips with the lateral thickness (or surface concentration) distribution of protein layers attached on a patterned surface. A ligand and its receptor such as an antibody and its corresponding antigen can assemble into complexes due to their affinity. The optical biosensor is based on that each reactant, referred to as a ligand, is immobilized to a surface area as a sensing surface with bioactivity of the ligand. The other reactant, as the analyte (or receptor), exists in solution. The sensing surface is exposed to the solution containing analyte. When the analyte in the solution interacts with its corresponding ligand on the sensing surface and assembles into complex upon their affinity. The layer on the surface where the interaction takes place becomes thicker (or surface concentration higher) than before the exposure to the analyte solution. A significant increase of the attached layer thickness (or surface concentration) indicates that the solution contained receptor against the ligand. With a visualization of imaging ellipsometry, the variation is determined, and in this way, the existence of the analyte in the solution can be verified. For the optical proteinchip, the substrate of the chip is treated by photolithography or "soft" lithography technique in order to create patterned surface $[3,4]$. Each area of the pattern is corresponding to one bioactivity with ligand immobilization, such as protein immobilization, and the interval between the areas is non-bioactive and optically extinguished. In this case, each area of the protein-chip may function as a biosensor for corresponding analyte (or receptor). 
The advantages of the protein-chip is: 1) a multi-detection performed at the same time; 2) the detection without labeling requirements; 3) in-situ and ex-situ detection available for protein analysis; 4) less consume of analytes; 5) quantitative detection with calibrations. Especially it is ideal way to monitor the protein adsorption process and the binding of proteins. Its function of real-time analysis for protein interaction may obtain some important kinetic data such as the protein interaction rate and the in-situ interaction conditions, etc. It is not only for one couple of proteins, but also for multicouples available at the same time.

\section{Methodology}

The micro-system includes several sections: 1) the chip design, which depends on the analytes which types and quantity should be considered; 2) the surface patterning, the chip substrate is patterned with photolithography or soft lithography; 3) surface modification in order to create specific surface properties for oriented ligand immobilization and block of unspecific binding; 4) ligand immobilization, to produce a sensing surface with assembling of ligand; especially the oriented immobilization with low steric hindrance and high bio-specific binding activity between ligand and receptor 5) the cell for protein interaction. Furthermore, the sampling, processing and analyzing of the results of protein interactions were performed with the optical biosensor system, respectively.

The sampling system with imaging ellipsometry has been mentioned in details before [5]. It has the ability to visualize the submonolayer of proteins, and the thickness resolution reaches to the order of $0.1 \mathrm{~nm}$.

The evolution of the chip is demonstrated in Fig.1. (A) A solid substrate of the biochip is prepared with the technique of photolithograph, similar to which used for micro-electronics elements, to pattern areas and each area isolated with shading background that is non-bioactive and optically extinctive. Each area surface of the pattern is atom-graded flat like a mirror, and its surface is chemically modified for ligand immobilization. (B) The ligand is immobilized with adsorption or self-assembly to form a sensing surface and the binding sites of ligand exposing to the upside. Various kinds of ligands are pre-attached on each area individually to form a bioactive pattern with many sensing areas one by one on the proteinchip surface. (C) After the interaction between proteins, the molecule complexes appear on the chip surface corresponding to analytes in the solution tested. (D) The sampling is performed with the imaging system of in-situ or ex-situ availability. The results are finally grabbed and stored in a computer for further analysis.

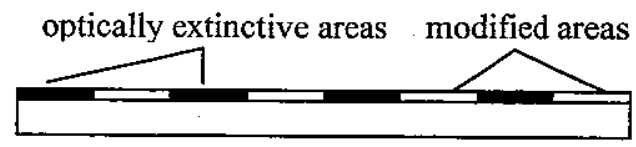

(A)

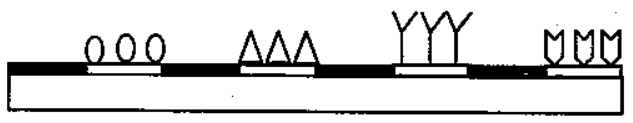

(B)

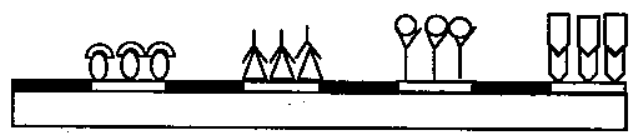

(C)

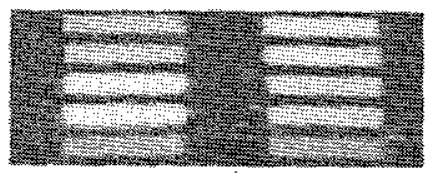

(D)

Fig.1. Proteinchip model

For the in-situ sampling, the interaction process between the analytes in solution and the ligands on the surface of patterned biochip can be visualized in real-time reaction cell specially designed. The cell has a small volume containing the solution and two optical windows for the probe light beam of imaging in and out. The normal of the two windows is parallel to the incident direction and the reflection direction of the probe light. The windows with optical quality are transparent for the incident light. The solution could be poured into the cell and pumped out by a micro-fluidics system with no disturbance for the optical probe. 
The chip is installed in the cell. When the solution containing the analytes is poured into the cell, the analytes in the solution will react with their corresponding ligands on the area surface of the chip according to the affinity of protein interaction, so that the thickness (or the surface concentration of protein) of sensing layer in patterned areas increase. The variation process of the layer thickness (or the surface concentration) can be grabbed in a series of images with time, furthermore it is possible to obtain kinetic information for the interactions from a digital image processing.

\section{Results and discussion}

\subsection{Protein-Chip for multi-protein interactions}

A silicon wafer surface was treated with photolithography into ten square areas (1.5 x 1.5 $\mathrm{mm}^{2}$ ) separated by the width of $1 \mathrm{~mm}$ of shading background. It was modified with dichlordimethylsilane to prepare for ligand immobilization. There were four kinds of proteins bovine serum albumin (BSA), human fibrinogen (Fib), human immunoglobulin G (IgG), and human serum albumin (HSA) in duplicate immobilized in the areas individually, which occupied eight areas and other two bares left as references.

The images sampled are shown in light intensity distribution, which correspond to the sensing layer thickness distribution or the surface concentration of proteins. It is capable of proving

$$
\operatorname{Th}(x, y, t) \propto \sqrt{I(x, y, t)}
$$

that the thickness $T h(x, y . t)$ of the protein layer is proportional to the square root of the intensity $\mathrm{I}(\mathrm{x}, \mathrm{y}, \mathrm{t})[6]$. In this way, the thickness distribution can be shown in three dimensions. The image in Fig. 2. (A) shows the thickness distribution of the protein pattern on the chip. The thickness of the HSA layer is almost the same as that of the BSA layer, owing to their similar molecular sizes and same immobilization conditions, the IgG's thicker and the Fib's the thickest. The entire sensing areas of the protein-chip were incubated in serum containing a mixture of antiserum, which contained antibodies of antiIgG, antiHSA and antiFib, etc. They reacted with their surfacebound antigens to form complexes IgG-antiIgG, HSA-antiHSA and Fib-antiFib, respectively, so that significant increases of layer thickness in the three couples of areas appeared. These can be obviously seen in Fig. 2 (B), which shows an image taken after the incubation in the antiserum. No significant thickness increase occurred in the BSA areas. Otherwise the sensing surface was incubated in the single antiserum containing antiIgG, anti-HSA, anti-BSA or anti-Fib, respectively, the corresponding images after incubations in antiserum confirmed the selectivity of such kind of protein-chip.

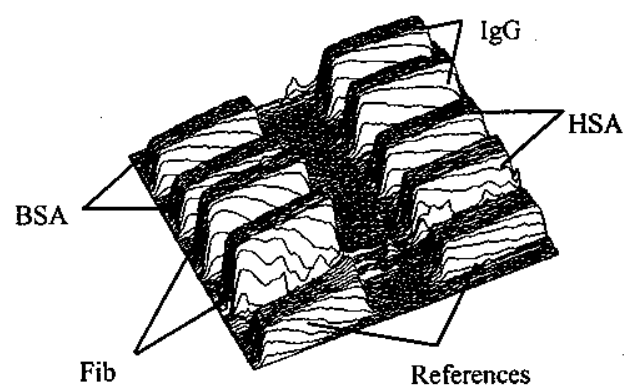

(A)

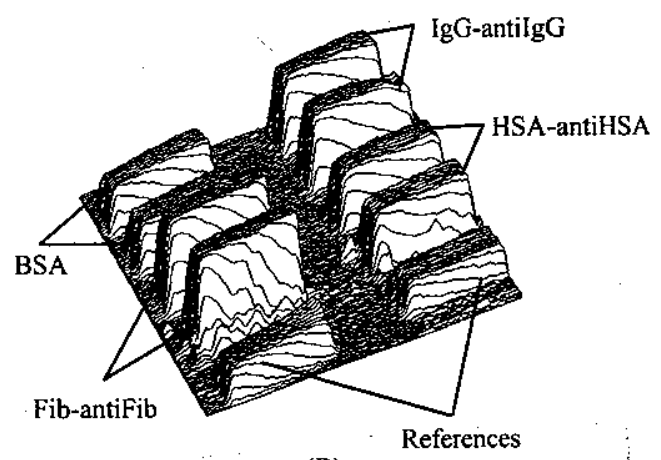

(B)

Fig. 2.(A) the chip with BSA, Fib, IgG and HSA layers (B) the chip after incubation in the serum with antiFib, antiIgG and antiHSA.

\subsection{Real-time visualization for multi-process of protein interactions}

On above, the final results for three couples of protein binding according to their affinities were observed on the protein-chip. Furthermore, the kinetic processes of protein interactions became into our interests. For the purpose of real time observation of multi-protein interaction processes, 
a similar chip with four kinds of immobilized ligand was prepared on the patterned surface. The chip was inserted into the cell containing solution for in-situ experiments and the chip surface with sensing areas was clearly observed. Then the mixture of antiserum containing antibodies of anti-IgG, anti-HSA and anti-Fib, etc. was poured into the cell. Several binding processes between antigens on the surface and antibodies in the solution were observed simultaneously. The binding processes resulted in the layer thickness on the corresponding sensing areas increasing with time. Fig. 3 . just shows a series of thickness variation with time for protein binding processes. These were deduced from the average thickness of corresponding sensing areas during the in-situ experiments. The relationship between the intensity $I(x, y . t)$ and the thickness $T h(x, y, t)$ of protein layer was followed, so the thickness variation means the protein binding to the surface on their affinity. Further observation showed that the binding rate of different protein couple was different from others.

The statistical average thickness (in arbitrary

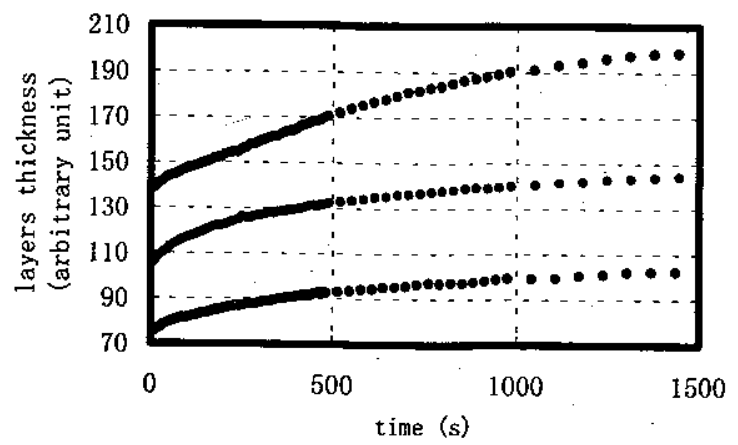

Fig. 3. Fib-antiFib, IgG-antiIgG and HSA-antiHSA binding processes

unit) over the IgG, HSA and Fib areas in realtime visualization are given in Fig. 3. There are two interaction rates for the three binding processes seen obviously below and over $100 \mathrm{~s}$.

When the solution with antisera was poured into the reaction cell, the antibody molecules diffused to everywhere of the cell to reach a uniform distribution of molecule concentration owing to the concentration diffusion. Some antibody molecules diffusing to the sensing surface reacted with the corresponding ligands on the surface binding into their complexes. This was corresponding to the process below $100 \mathrm{~s}$. The capital phenomenon is the diffusion rate that controlled the binding rate. Then the concentration distribution of antibody in the cell reached to a uniformity. The interaction with a higher rate occurred on whole the sensing surface over $100 \mathrm{~s}$. It showed that the interaction rate is different among the three binding processes. The binding rate between Fib and antiFib is faster than the others, and the slowest one is between HSA and its antibody. Here, only a sketchy description for the protein interaction processes simultaneously detected is presented as a demonstration. The further theoretical analysis in details is presented in another paper.

\section{Conclusion}

The above demonstration of the proteinchip micro-system for protein interactions shows a potential way for multi-protein analysis. It has advantages in simple operation, no labeling, and simultaneous multi-channel analysis. It's suitable for micro-assays related to various specific bindings between bio-molecules.

\section{Acknowledgments}

The Chinese Academy of Sciences is acknowledged for its supports.

\section{References}

[1] G. JIN, R. Jansson, I. Lundstrom and H. Arwin, "Imaging ellipsometry for biosensor applications", Transducers'95 Eurosensors IX, Stockholm, Sweden, June, 1995.

[2] G. JIN, P. Tengvall, I. Lundstrom and H. Arwin, "A Biosensor Concept Based on Imaging Ellipsometry for -Visualization of Biomolecular Interactions", Analytical Biochemistry,.232,( 1995) 69-72

[3] R. S. Kane, et al., "Patterning proteins and cells using soft lithography", Biomaterials, 20(23-24)( 1999) 2363-2376

[4] H. Fan, et al., "Rapid prototyping of patterned functional nanostructures," Nature, 405 $(6782)(2000) 56-60$

[5] G. JIN, R. Jansson and H. Arwin, "Imaging Ellipsometry Revisited: Developments for Visualization of Thin Transparent Layers on Silicon Substrates," Rev. Sci. Instrum.,67(1996)2930-2936 [6] H. Arwin, S. Welin, and R. Jansson, J. Colloid Interface Sci., 156(1993)377 